\title{
What Variability of Treatment Effect of Fampridine Can We Expect in
}

\section{People with Multiple Sclerosis?}

\section{Klara Novotna $^{1 *}$, Jana Lizrova Preiningerova ${ }^{1}$, Lukas Sobisek ${ }^{2}$ and Eva Kubala Havrdova ${ }^{1}$}

${ }^{1}$ Department of Neurology and Center of Clinical Neuroscience, First Faculty of Medicine, Charles University and General University Hospital, Prague, Czech Republic ${ }^{2}$ Department of Statistics and Probability, University of Economics, Prague, Czech Republic

\section{Abstract}

Background: Gait impairment represents one of the most common symptoms of multiple sclerosis (MS). Fampridine is the first symptomatic treatment aimed at improving gait.

An objective measurement of the mobility improvement from treatment initiation has been recommended to evaluate treatment response.

Objective: In this retrospective observational study, we evaluated what improvement in walking speed can be expected in people with multiple sclerosis (MS) treated with Fampridine in clinical practice, with respect to specific disability levels (EDSS 4.0-7.0).

Methods: The mobility tests including the Timed 25 foot walk test (T25FW), Timed Up and Go test (TUG) and Step test (ST) were performed just before and $3 \mathrm{~h}$ after administration of Fampridine $10 \mathrm{mg}$ tablet.

Results: One hundred and thirty one (131) people with MS (15 with primary progressive, 40 with secondary progressive and 76 people with relapsing-remitting MS). The mean age was 48 years (SD 9.8), mean MS duration was 19, 8 years, $58 \%$ were women. The range of treatment response of Fampridine, measured with the T25FW test, varied from $11-41 \%$.

Contrary to prior reports, the baseline T25FW and the percentage of improvement in T25FW was significantly correlated.

Conclusion: Assessment of treatment response outside of a clinical trial is challenging and may require different outcome measures compared to RCT. For MS patients with moderate disability seems TUG test or Step test more appropriate for quantifying treatment response.

Keywords: Multiple sclerosis; Gait; Disability; Symptomatic treatment; Fampridine

\section{Introduction}

Multiple sclerosis (MS) is an autoimmune inflammatory condition that causes myelin and axonal damage in the central nervous system, and can lead to disability in young adults. Symptoms of MS may include a variety of symptoms, such as muscle weakness, sensory dysfunction, fatigue, spasticity, ataxia, tremor, pain or visual impairment, and most people with MS suffers some degree of gait impairment, considered to be the most valuable bodily function in these individuals [1].

Fampridine is a potassium channel blocker that restores signal conduction of demyelinated fibers in patients with MS. The Fampridine $10 \mathrm{mg}$ prolonged release (FPR) tablet was approved in 2011 by the European Medicines Agency for improving of walking in adult MS patients with walking disability (Expanded Disability Status Scale 4.0-7.0) [2]. Not all patients experience benefits from treatment with FPR, but there no known markers to predict the treatment response. It is recommended that a timed walked test be performed to measure improvement in walking speed.

In the pivotal studies, MS-F203 and MS-F204, the primary outcome was defined as the percentage of responders (responder defined as a patient whose walking speed determined by the Timed 25 Foot Walk test (T25FW) at least three visits during the treatment period, was faster than the maximum speed measured in the five off-treatment visits). The average proportion of patients treated with FPR that meet this endpoint was $38.8 \%$ ( $34.8 \%$ vs. $8.3 \%$ in MS-F203 and $42.9 \%$ vs. $9.3 \%$ in MSF204). Other studies have also reported similar proportion of responders $[3,4]$. The average improvement in walking speed of patients in the treated groups of both MS-F203 and MS-F204 was $26.3 \%$ and $25.3 \%$ respectively. This improvement was apparent at the first treatment visit, which was scheduled 2 weeks after initiation of treatment $[5,6]$.
Identification of responders in clinical practice is difficult and so far there are no known markers predicting the response to this medication. Goodman et al. demonstrated that patient response to FPR treatment is independent of their demographic and clinical characteristics and also concluding that the percent improvement is independent of their disability level and baseline walking speed [7]. Pivotal trials conducted by Goodman et al. [7] limited patient enrollment to those with a walking speed of between 8 to $45 \mathrm{~s}$ in the T25FW test.

In this retrospective observational study, we evaluated the extent of improvement in the T25FW test that can be expected in patients treated with FPR in clinical practice, considering specific disability levels (EDSS 4.0-4.5, EDSS 5.0-5.5, EDSS 6.0, EDSS 6.5 and EDSS 7.0).

Day to day variability in mobility is common in people with MS [8]. In order to limit factors that may confound the measurement of walking speed, the functional test of response to FPR were performed on the same day, immediately prior to administration of the first dose and then $3 \mathrm{~h}$ later. The maximum plasma concentration for FPR is reached 3.3-3.9 h post administration [9].

*Corresponding author: Novotna K, Department of Neurology and Center of Clinical Neuroscience, First Faculty of Medicine, Charles University and General University Hospital, Prague, Czech Republic, Tel: 00420224966524; Fax: 00420917907; E-mail: novotna.klara.k@gmail.com

Received July 11, 2017; Accepted July 28, 2017 ; Published August 04, 2017

Citation: Novotna K, Preiningerova JL, Sobisek L, Havrdova EK (2017) What Variability of Treatment Effect of Fampridine Can We Expect in People with Multiple Sclerosis? J Mult Scler (Foster City) 4: 206. doi: 10.4172/2376-0389.1000206

Copyright: () 2017 Novotna K, et al. This is an open-access article distributed under the terms of the Creative Commons Attribution License, which permits unrestricted use, distribution, and reproduction in any medium, provided the original author and source are credited 


\section{Methods}

\section{Subjects}

In this study, we included patients with MS that were prescribed FPR between January 2013 and April 2014. Treatment with FPR was indicated by the patient's treating physicians (MS specialists practicing in a large academic MS center) based on the patient's needs and an evaluation of gait by a physical therapist was requested. FPR has been approved in our clinic for MS patients with EDSS 4.0-7.0 and the cost is not covered by health insurance companies. Patients did not receive any incentive for participating in this evaluation, other than results from the measurement, and patients covered the cost of the medication themselves. All participants signed informed consent form and the study was approved by the Ethical Committee of General University Hospital in Prague. Patients were excluded if they showed MS exacerbation within 60 days of treatment initiation, a history of seizure or any condition that would interfere with walking evaluation (injury, orthopedic problems, etc.).

\section{Procedure}

Level of disability in people with MS is described by Expanded Disability Status Scale (EDSS) [10]. Baseline EDSS was performed by an MS specialist. Patients completed the MSWS12 questionnaire. The mobility tests including Timed 25 foot walk test (T25FW test), Timed up and go test (TUG test) and step test (ST), which were performed by a physical therapist just before (baseline) and $3 \mathrm{~h}$ after oral administration of a fampridine tablet $(10 \mathrm{mg})$.

The T25FW is a standardized walking test that forms part of the Multiple Sclerosis Functional Composite (MSFC) test, which measures the walking speed of the patient over 25 feet $(7.62 \mathrm{~m})$ [11]. The TUG test is a standard mobility test that measures the time for a subject to get up from sitting position, walk $3 \mathrm{~m}$, then turn and walk back to sit on chair [12]. The ST was performed with one item modified from the Berg Balance test. Briefly medical step stool $(25 \mathrm{~cm}$ high) was used to count how many times the patient, positioned standing in front of the stool (patients with EDSS 6.5 and higher used support), was able to touch the step with one foot over a period of $30 \mathrm{~s}[13,14]$.

The patient were asked to subjectively evaluate perceived effect of the medication on their physical well-being using a 5-point scale, where 5 represented="very much improved" and 1 represented="feeling worse".

\section{Statistical analysis}

Descriptive statistics (mean and standard deviation (SD) of the characteristics of interest were calculated for each patient and by subgroups (by gender, MS phenotype and EDSS). Correlation analysis was carried out using Spearman's rank correlation coefficient. Pairwise comparison of the absolute change in step test was carried out using Tukey and Kramer test with Tukey-Dist approximation for independent samples. All analyses were performed using statistical software $\mathrm{R}$ (http://www.r-project.org/).

\section{Results}

This analysis included 131 MS patients. There were 15 patients with primary progressive MS, 40 with secondary progressive MS and 76 patients with a relapsing-remitting form of MS. The mean age of patients was 48 years (SD 9.8), the mean MS duration was 19.8 years (SD 8.6) and 58\% were women. The mean MSWS-12 score was 46 (Table 1).

Of the 131 patients 44 participants (34\%) reached at least $20 \%$ improvement in $\mathrm{T} 25 \mathrm{FW}$ and were considered responders.

\section{Mobility tests}

The response to treatment, presented as the mean change from baseline in all three mobility tests according to patient EDSS level (Figure 1). The average improvement of speed in the T25FW test was $11 \%$ in patients with EDSS 4.0, patients with EDSS 4.5, EDSS 5.0 and EDSS 5.5 showed $15 \%, 19 \%$ and $20 \%$ improvement, respectively and those with EDSS 6.0, EDSS 6.5 and EDSS 7.0 improved by 19\%, 18\% and $23 \%$, respectively (Table 2 ).

Of the 131 patients, 44 participants (34\%) reached at least $20 \%$ improvement in the T25FW test. If $20 \%$ improvement in the T25FW test was considered clinically significant consistent with several MS gait studies $(15,16)$, this would indicate that only 1 out of 19 patients (5\%) with EDSS 4.0 meet this threshold, whereas $41 \%$ and $40 \%$ of patients with EDSS 6.0 and EDSS 6.5, respectively reached this level of improvement (Table 2).

The relationship between the baseline T25FW and the percentage of change in T25FW due to the treatment (all patients were included in the analysis regardless of their subjective response). There was a significant correlation between baseline T25FW and percentage of improvement in $\mathrm{T} 25 \mathrm{FW}$ (Spearman rank correlation, $\mathrm{rho}=0.60, \mathrm{p}<0.0001$ ).

Not all patients with higher EDSS level were able to perform the TUG and ST tests due to neurological impairment (4/10 patients with EDSS 7.0 and 27/30 patients with EDSS 6.5 completed the test). The average improvement in the TUG test in patients in groups EDSS 4.07.0 ranged from $14 \%$ to $22 \%$, shown in Table 3 . When we compared how the three mobility tests reflected the treatment response, patients with EDSS 6.0-6.5 who showed 20\% improvement in T25FW were also

\begin{tabular}{|c|c|c|c|c|}
\hline Number of participants & $\begin{array}{l}\text { Age (years) } \\
\text { Mean (SD) }\end{array}$ & $\begin{array}{c}\text { EDSS } \\
\text { Mean (SD) }\end{array}$ & $\begin{array}{l}\text { Disease duration } \\
\text { Mean (SD) }\end{array}$ & $\begin{array}{c}\text { MSWS } 12(12-60) \\
\text { Mean (SD) }\end{array}$ \\
\hline Total: N=131 & $48.06(9.82)$ & $5.7(0.93)$ & $20.15(8.4)$ & $46.18(8.05)$ \\
\hline Female: $\mathrm{N}=76$ & $47.5(9.93)$ & $5.68(0.94)$ & $19.56(7.30)$ & $44.99(7.97)$ \\
\hline Male: $\mathrm{N}=55$ & $48.84(9.7)$ & $5.73(0.92)$ & $20.94(9.64)$ & $47.82(7.86)$ \\
\hline Relapsing-remitenting: $\mathrm{N}=76$ & $45(8.61)$ & $5.41(1.02)$ & $18.09(7.36)$ & $44.47(8.54)$ \\
\hline Secondary progressive: $\mathrm{N}=40$ & $53.17(9.68)$ & $6.09(0.54)$ & $23.82(8.47)$ & $48.13(6.51)$ \\
\hline Primary progressive: $\mathrm{N}=15$ & $49.93(10.39)$ & $6.13(0.74)$ & $20.37(9.49)$ & $49.31(6.99)$ \\
\hline EDSS 4-4.5 N=29 & $44.55(7.82)$ & $4.17(0.23)$ & $16.32(7.82)$ & $36.41(6.89)$ \\
\hline EDSS $5-5.5 \mathrm{~N}=16$ & $51.18(9.85)$ & $5.31(0.24)$ & $20(10.28)$ & $47(6.07)$ \\
\hline EDSS $6 \mathrm{~N}=46$ & $49.47(9.94)$ & $6(0)$ & $20.23(6.97)$ & $47.78(6.08)$ \\
\hline EDSS $6.5 \mathrm{~N}=30$ & $49.7(9.56)$ & $6.5(0)$ & $24.13(9.07)$ & $50.07(4.56)$ \\
\hline EDSS $7 \mathrm{~N}=10$ & $41.8(8.37)$ & $7(0)$ & $18.8(4.48)$ & $54.1(4.67)$ \\
\hline
\end{tabular}

Table 1: Demographic characteristics of participants. 


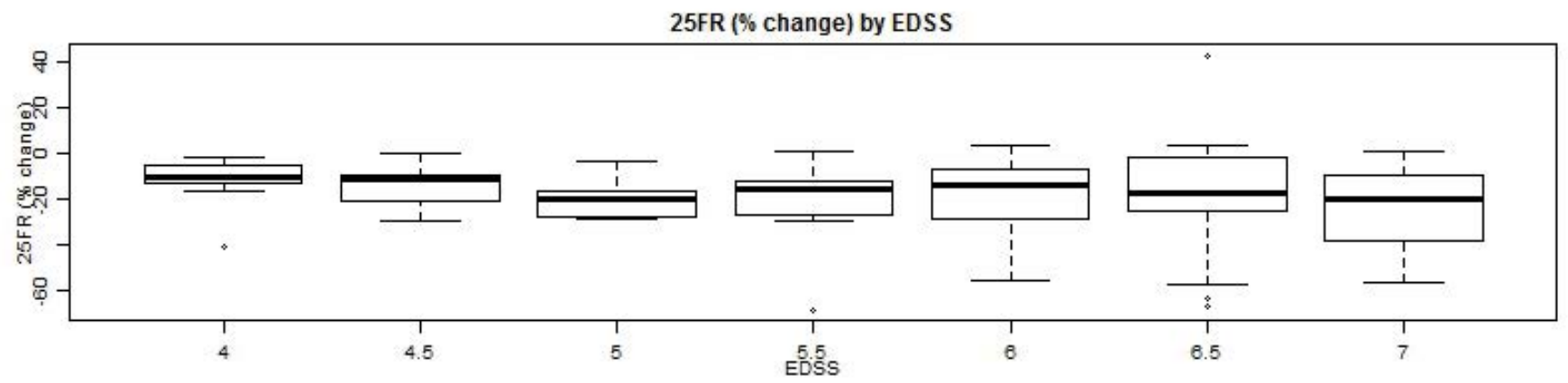

TUG (\% change) by EDSS

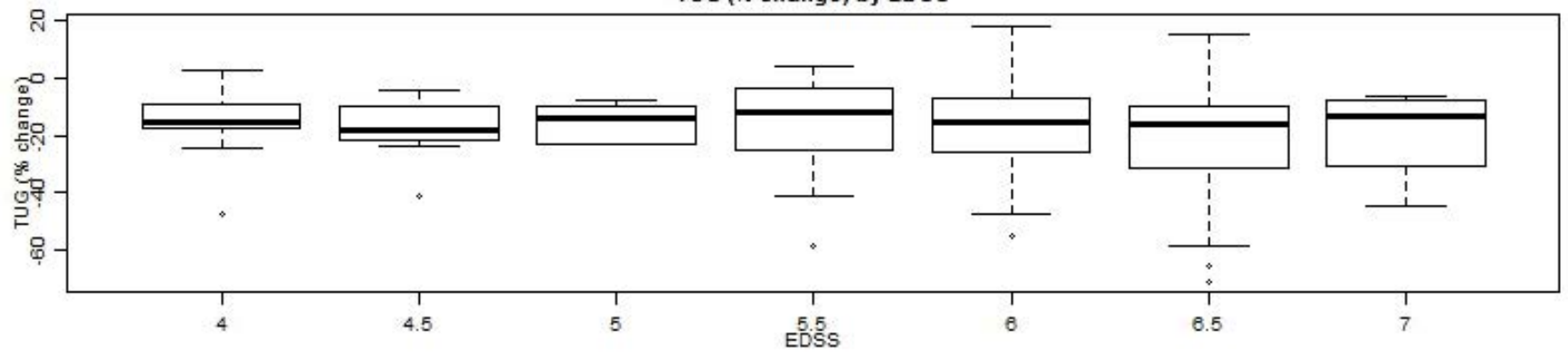

schod (abs change) by EDSS

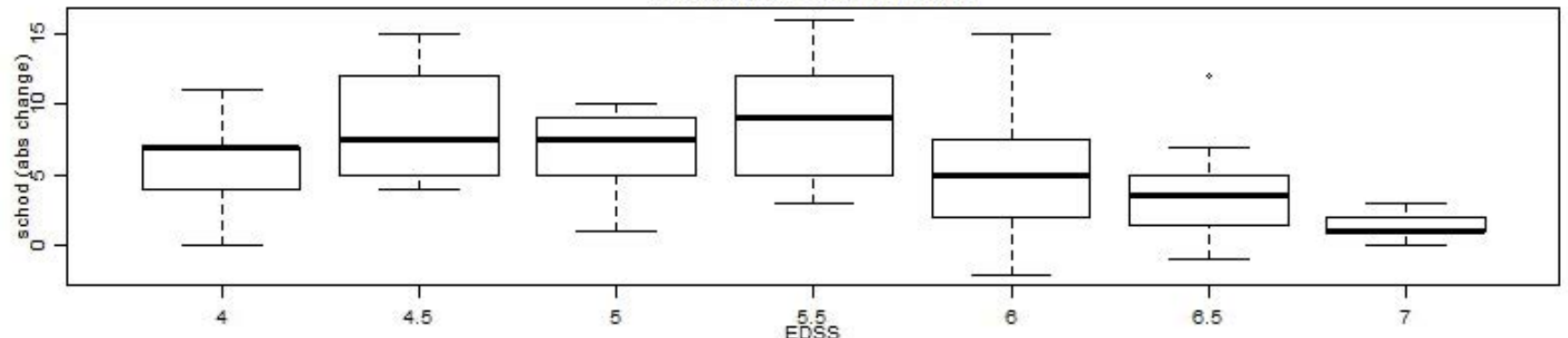

Figure 1: Three mobility tests according to patient EDSS level.

\begin{tabular}{|c|c|c|c|c|c|}
\hline EDSS & $\begin{array}{l}\text { Mean T25FW-baseline } \\
\text { (seconds, s) }\end{array}$ & $\begin{array}{l}\text { Mean T25FW-on medication } \\
\text { (seconds, s) }\end{array}$ & T25FW-\% change & Improvement $\geq 20 \%$ & Improvement $\geq 15 \%$ \\
\hline $4(\mathrm{~N}=19)$ & 5.9 (SD 1.62) & 5.18 (SD 1.09) & 10.77 (SD 8.66) & $\begin{array}{c}N=1 \\
5.26 \%\end{array}$ & $\begin{array}{c}\mathrm{N}=2 \\
10.52 \%\end{array}$ \\
\hline $4.5(\mathrm{~N}=10)$ & 7.01 (SD 1.6) & 5.91 (SD 1.12) & 14.67 (SD 8.89) & $\begin{array}{l}N=2 \\
10 \%\end{array}$ & $\begin{array}{l}N=2 \\
10 \%\end{array}$ \\
\hline $5(N=6)$ & 9.4 (SD 3.34) & 8.93 (SD 5.96) & 19.45 (SD 9.41) & $\begin{array}{l}N=3 \\
50 \%\end{array}$ & $\begin{array}{c}\mathrm{N}=2 \\
33.3 \%\end{array}$ \\
\hline $5.5(\mathrm{~N}=10)$ & 10.92 (SD 9.45) & 7.28 (SD 2.55) & 20.27 (SD 19.33) & $\begin{array}{l}N=3 \\
30 \%\end{array}$ & $\begin{array}{l}N=2 \\
20 \%\end{array}$ \\
\hline $6(\mathrm{~N}=46)$ & $15.4(S D 18.54)$ & $10.92(S D 8.24)$ & 18.83 (SD 14.99) & $\begin{array}{l}\mathrm{N}=19 \\
41.3 \%\end{array}$ & $\begin{array}{c}\mathrm{N}=4 \\
8.69 \%\end{array}$ \\
\hline $6.5(\mathrm{~N}=30)$ & $25.4(S D 21.31)$ & 19.21 (SD 14.84) & 18.04 (SD 22.34) & $\begin{array}{l}N=12 \\
40 \%\end{array}$ & $\begin{array}{l}N=6 \\
20 \%\end{array}$ \\
\hline $7(\mathrm{~N}=10)$ & 71.5 (SD 51.05) & 53.89 (SD 39.02) & 23.52 (SD 18.67) & $\begin{array}{l}N=5 \\
50 \%\end{array}$ & $\begin{array}{l}N=1 \\
10 \%\end{array}$ \\
\hline
\end{tabular}

Table 2: Performance in T25FW.

likely to show improvement in the TUG test. Functional improvement in the response to treatment in patients with EDSS 4.0 and 4.5 was more apparent in the ST test than in the T25FW test (Figure 1).

There is evidence of a strong positive linear relationship between the relative change in T25FW and the relative change in TUG $(r=0.68$, $\mathrm{p}<0.0001)$. There was no statistically significant correlation between the change in mobility tests and the patients age or change in T25FW and age $(\mathrm{p}=0.957)$ and change in T25FW and EDSS ( $\mathrm{p}=0.623)$, no significant difference in the absolute change in the ST $(\mathrm{p}=0.847)$ between patients with EDSS $4.0(\mathrm{n}=19$, median=7) and patients with EDSS $5.5(\mathrm{n}=10$, median=9). There was a significant decrease in absolute change in the ST when patients with EDSS 5.5 were compared to both groups of patients with higher impairment, particularly the group of patients with EDSS $6.5(\mathrm{n}=28$, median $=3,5, \mathrm{p}=0.015)$ and patients with EDSS $7.0(\mathrm{n}=5$, median $=1, \mathrm{p}=0.018)$. 


\begin{tabular}{|c|c|c|c|c|}
\hline $\begin{array}{c}\text { EDSS Number of } \\
\text { participants }\end{array}$ & $\begin{array}{c}\text { Mean TUG-baseline } \\
\text { (seconds, s) }\end{array}$ & $\begin{array}{c}\text { Mean TUG-on medication } \\
\text { (seconds, s) }\end{array}$ & $\begin{array}{c}\text { TUG-\%change } \\
\text { Step test-baseline } \\
\text { (number of steps) }\end{array}$ & $\begin{array}{c}\text { Step test-on medication } \\
\text { (number of steps) }\end{array}$ \\
\hline $4(\mathrm{~N}=19)$ & $8.12(2.14)$ & $6.86(1.6)$ & $14.49(10.2)$ & $19.21(6.61)$ \\
\hline $4.5(\mathrm{~N}=10)$ & $9.95(1.42)$ & $8.11(1.33)$ & $17.98(10.18)$ & $13.3(6.02)$ \\
\hline $5(\mathrm{~N}=6)$ & $12.13(3.1)$ & $10.22(2.43)$ & $15.25(6.65)$ & $21.5(6.19)$ \\
\hline $5.5(\mathrm{~N}=10)$ & $13.48(7.39)$ & $10.17(3.32)$ & $18.07(19.87)$ & $20.67(3.98)$ \\
\hline $6(\mathrm{~N}=44)$ & $16.7(8.07)$ & $13.12(4.98)$ & $18.13(13.9)$ & $9.5(5.54)$ \\
\hline $6.5(\mathrm{~N}=27)$ & $34.81(33.17)$ & $24.27(22.19)$ & $22.97(20.14)$ & $18.5(5.82)$ \\
\hline $7(\mathrm{~N}=4)$ & $29.27(7.27)$ & $22.6(3.73)$ & $14.24)$ & $6.71(5.27)$ \\
\hline
\end{tabular}

Table 3: Performance in mobility tests (TUG and step test).

\begin{tabular}{|c|c|c|c|c|c|}
\hline EDSS & 5-very much improved & 4-much improved & 3-improved & 2-no effect & 1 -worse \\
\hline $4-4.5(\mathrm{~N}=29)$ & $4(13.8 \%)$ & $16(55.1 \%)$ & $5(17.24 \%)$ & $4(13.8 \%)$ & $0(0 \%)$ \\
\hline $5-5.5(\mathrm{~N}=16)$ & $0(0 \%)$ & $10(62.5 \%)$ & $2(12.5 \%)$ & $3(18.75 \%)$ & $1(6.25 \%)$ \\
\hline $6(N=46)$ & $2(4.34 \%)$ & $25(54.34 \%)$ & $10(21.73 \%)$ & $7(15.21 \%)$ & $2(4.34 \%)$ \\
\hline $6.5(\mathrm{~N}=30)$ & $3(10 \%)$ & $9(30 \%)$ & $6(20 \%)$ & $11(3.66 \%)$ & $1(0.33 \%)$ \\
\hline $7(N=10)$ & $0(0 \%)$ & $5(50 \%)$ & $3(30 \%)$ & $2(20 \%)$ & $0(0 \%)$ \\
\hline
\end{tabular}

Table 4: Subjective evaluation of treatment effect on mobility.

\section{Patient-reported effect}

Of the patients, 74 (57\%) reported subjective improvement from treatment by choosing a score of 5 (very much improved) or 4 (much improved) on the 5 point scale. Among the remaining participants, $33(25 \%)$ were not sure of meaningful improvement (score 3 ) and 24 patients (18\%) reported a score of 2 (no change) or 1 (feeling worse) 3 $\mathrm{h}$ post treatment. Subjective evaluation of the effect, separated by EDSS level (Table 4).

\section{Discussion}

Not all patients experience benefits from treatment with FPR, and treatment response cannot be predicted. Therefore, regulatory agencies have recommended verification of gait improvement within 2 weeks of treatment initiation. This study, reporting our experience with FPR treatment in a clinical setting, has raised several important issues related to the assessment of treatment responses outside of a clinical trial.

While randomized placebo-controlled studies (RCT) are the best available tool for providing evidence of treatment efficacy, extrapolation of the RCT methods into clinical practice is challenging. In addition, clinical trials limit enrollment to a carefully selected population, which is generally not representative of the wide variety of patients that use FPR in clinical practice. Our study included patients within the indication for FPR established by the European Medicine Agency (EDSS 4.0-7.0) that had a baseline T25FW between 4, 12 and $181 \mathrm{~s}$, therefore our study provides data on the treatment response of patients with a wider disability range than that provided by clinical trials (MSF203 and MS-F204 limited enrollment by baseline T25FW between 8 and $45 \mathrm{~s})$.

The efficacy outcome measures of the MS-F203 and MS-F204 studies was based on repeated measures of the T25FW time. Analysis of pivotal trials data by Hobart et al. revealed, that improvements in T25FT of greater than $20 \%$ is associated with a clinically meaningful change in self-reported walking ability (MSWS12) [15]. Another study confirmed a $20 \%$ change in T25FW as being clinically meaningful, correlated with Physical Component Summary score of the 36-Item Short Form Health Survey [16]. A pooled analysis of the pivotal phase 3 studies, including patients with baseline T25FW between 8 to $45 \mathrm{~s}$, lead to conclusions that the responder rate was independent of the baseline walking speed [7]. This has set the expectation for clinical practice, and also for some payers assessing the efficacy of FPR treatment in individual's patients.
We demonstrated a significant correlation between baseline T25FW and the percentage of improvement in T25FW with FPR treatment. As shown in Table 2, only 1\% (3/29) of patients with EDSS 4.0-4.5 reached $>20 \%$ improvement in the T25FW tests, whereas the proportion of EDSS 5.0 patients reaching $>20 \%$ improvement in T25FW was higher, with at least $37 \%$ reaching this level of improvement. Of note, patients with EDSS 4.0 and 4.5 had mean T25FW times of 5.9 and $7.0 \mathrm{~s}$, respectively, and a $20 \%$ improvement in T25FW would bring them to 4.7 and 5.6 $\mathrm{s}$, which would be close to exceeding what a healthy individual of their age could be expected to achieve. There was no difference in the percentage of patients that reported subjective improvement from treatment with FPR across EDSS 4.0-7.0. We suspect that the potential for T25FW improvement in patients with EDSS 4.0 and 4.5 reaches a plateau, therefore, the functional improvement perceived by these patients should be measured by different test.

Although the mean change in T25FW was only $11 \%$ in patients with EDSS 4.0, the positive effect of the medication should be considered as being clinically meaningful, as T25FW time of over $6 s$ is associated with the requirement for greater assistance in daily activities, such as the use of cane for walking outside [17]. This is further supported by findings that a subjective perceived improvement in walking is associated with an improvement in normal work and daily activities, as well as the individual's social life [18]. A recent study has shown that responses to FPR were able to increase their physical activity in everyday life [19].

\section{Other functional measures}

A change in TUG was found to be strongly correlated with a change in T25FW, however, the TUG test is known to be more physically challenging. In patients with EDSS 4.0 and 4.5, the mean relative improvement in TUG was higher (14.5\% and $18 \%$, respectively) than the mean improvement in $\mathrm{T} 25 \mathrm{FW}$ ( $11 \%$ and $15 \%$, respectively). Improvement of performance in the TUG test demonstrates the effect of FPR on dynamic balance and standing from a sitting position. Our observation of an improvement in dynamic balance builds on the finding from a study by Prosperini et al. which showed an effect of FPR on static standing balance [6]. Improvement in TUG after FPR, when compared to a placebo, was also described in the MOBILE trial [20].

The ST, as performed by patients in our FPR assessment, remains exploratory, as this test has not been completely validated. Performance in the ST is dependent on the range of movement and is influenced by spasticity, muscle strength of the lower limbs and balance. Therefore, 
an improvement in this test indirectly shows improvements in these domains. Several studies have documented the positive effect of FPR on muscle strength of lower limbs and reducing spasticity in patients with MS $[9,17]$. In some responders to treatment, FPR can increase the range of motion in the knee or hip joint, having a positive impact on the walking pattern [19].

\section{Subjective assessment}

A high percentage of patients responded positively to the question of how they rated the perceived effect of the medication. In cannot be excluded that patients were hoping to be responders and the lack of blinding is a major limitation of this assessment. In addition, patients had a limited time for evaluating their response. The standard patientreported measure of the impact of MS on walking, such as the MSWS12 , cannot be used for same-day testing, but is more suitable for testing the effect of a particular treatment over time. Findings from a recent study demonstrated the importance of patient-reported outcomes in the evaluation of patient perspective and experience [18].

\section{Same day testing}

The choice of testing the effect of treatment on the same day was motivated by the need to eliminate variability in T25FW, caused by various factors known to affect performance in patients, physical exertion prior to gait testing, concurrent illness, and so on. These factors are controlled for in the RCT setting by careful monitoring of these testing conditions, as well as repeated testing, which was not feasible in our practice.

\section{Limitations of this study}

Interpretation of data derived from a retrospective study needs to consider the limitations of such studies. Interpretation of data is limited by the open-label nature of testing, where (neither patient nor evaluator could be blinded to treatment administration), which may account for the high proportion of patients reporting subjective improvement from FPR treatment. Another limitation of the study was that we did not include confirmatory assessment of walking within the days following treatment, or the effects of treatment over a longer period of time. Same day testing of treatment with FPR has not yet been validated and is therefore exploratory. The current clinical assessment does not provide information on the effects of FPR on non-mobility domains, although it has previously been reported that FPR affect hand function, cognition, depression, fatigue, quality of life and verbal fluency [21-25].

\section{Conclusion}

Assessment of treatment response outside of a clinical trial is challenging and may require different outcome measures compared to RCT.

\section{Acknowledgement}

This study was supported by a grant of Czech Ministry of Education Progres Q27/LF1. Statistical analysis was supported by a grant from Novartis.

\section{References}

1. Heesen C, Bohm J, Reich C, Kasper J, Goebel M, et al. (2008) Patien perception of bodily functions in multiple sclerosis: Gait and visual function are the most valuable. Mult scler 14: 988-991.

2. Mathiesen HK, Sorensen PS (2013) Prolonged-release fampridine improves walking in a proportion of patients with multiple sclerosis. Expert Rev Neurother 13: $1309-1317$

3. Goodman AD, Brown TR, Edwards KR, Krupp LB, Schapiro RT, et al. (2010) A phase 3 trial of extended release oral dalfampridine in multiple sclerosis. Ann Neurol 68: 494-502.
4. Goodman AD, Brown TR, Krupp LB, Schapiro RT, Schwid SR et al. (2009) Sustained-release oral fampridine in multiple sclerosis: A randomised, doubleblind, controlled trial. Lancet 28: 732-738.

5. Keune PM, Cocks AJ, Young WR, Burschka JM, Hansen S, et al. (2015) Dynamic walking features and improved walking performance in multiple sclerosis patients treated with fampridine (4-aminopyridine). BMC Neurol 15 171

6. Prosperini L, Gianni C (2014) Oral dalfampridine improves standing balance detected at static post-urography in multiple sclerosis 2014: 802307.

7. Goodman AD, Brown TR, Schapiro RT, Klingler M, Cohen R, et al. (2014) A pooled analysis of two phase 3 clinical trials of dalfampridine in patients with multiple sclerosis. Int J MS Care 16: 153-160.

8. Albrecht H, Wotzel C, Erasmus LP, Kleinpeter M, Konig N, et al. (2001) Dayto-day variability of maximum walking distance in MS patients can mislead to relevant changes in the expanded disability dtatus dcale (EDSS): Average walking speed is a more constant parameter. Mult Scler 7: 105-110.

9. Vollmer T, Henney HR, 3rd. Pharmacokinetics and tolerability of single escalating doses of fampridine sustained-release tablets in patients with multiple sclerosis: A Phase I-II, open-label trial. Clin Ther 31: 2206-2214.

10. Kurtzke JF (1955) A new scale for evaluating disability in multiple sclerosis Neurology 5: 580-583.

11. Kaufman M, Moyer D, Norton J (2000) The significant change for the timed 25foot walk in the multiple sclerosis functional composite. Mult Scler 6: 286-290.

12. Sebastiao E, Sandroff BM, Learmonth YC, Motl RW (2016) Validity of the timed up and go as a measure of functional mobility in persons with multiple sclerosis. Arch Phys Med Rehab 97: 1072-1077.

13. Berg KO, Wood-Dauphinee SL, Williams JI, Maki B (1992) Measuring balance in the elderly: Validation of an instrument. Can J Public Health 2: S7-S11.

14. Cattaneo D, Regola A, Meotti M (2006) Validity of six balance disorders scales in persons with multiple sclerosis. Disabil Rehabil 28: 789-795.

15. Cohen JA, Cutter GR, Fischer JS, Goodman AD, Heidenreich FR, et al. (2001) Use of the multiple sclerosis functional composite as an outcome measure in a phase 3 clinical trial. Arch neurol 58: 961-967.

16. Hobart J, Blight AR, Goodman A, Lynn F, Putzki N (2013) Timed 25-foot walk: Direct evidence that improving $20 \%$ or greater is clinically meaningful in MS Neurol 80: 1509-1517.

17. Goldman MD, Motl RW, Scagnelli J, Pula JH, Sosnoff JJ, et al. (2013) Clinically meaningful performance benchmarks in MS: Timed 25-foot walk and the real world. Neurol 81: 1856-1863.

18. Crayton H, Sidovar M, Wulf S, Guo A (2015) Patient perspectives and experience with dalfampridine treatment in multiple sclerosis-related walking impairment: The step together program. Patient 8: 283-291.

19. Zorner B, Filli L, Reuter K, Kapitza S, Lorincz L, et al. (2016) Prolonged-release fampridine in multiple sclerosis: Improved ambulation effected by changes in walking pattern. Mult Scler 22: 1463-1475.

20. Hupperts R, Lycke J, Short C, Gasperini C, McNeill M, et al. (2015) Prolonged release fampridine and walking and balance in MS: Randomised controlled MOBILE trial. Mult Scler 22: 212-221.

21. Jensen H, Ravnborg M, Mamoei S, Dalgas U, Stenager E (2014) Changes in cognition, arm function and lower body function after slow-release Fampridine treatment. Mult Scler 20: 1872-1880.

22. Lo AC, Ruiz JA, Koenig CM, Anderson BM, Olson KM, et al. (2015) Effects of dalfampridine on multi-dimensional aspects of gait and dexterity in multiple sclerosis among timed walk responders and non-responders. J Neurol Sci 356 $77-82$

23. Pavsic K, Pelicon K, Ledinek AH, Sega S (2015) Short-term impact of fampridine on motor and cognitive functions, mood and quality of life among multiple sclerosis patients. Clin Neurol Neurosurg 139: 35-40.

24. Prugger $M$, Berger $T$ (2013) Assessing the long-term clinical benefit of prolonged-release fampridine tablets in a real-world setting: A review of 67 cases. Patient Relat Outcome Meas 4: 75-85.

25. Magnin E, Sagawa Y, Chamard L, Berger E, Moulin T, et al. (2015) Verbal fluencies and fampridine treatment in multiple sclerosis. Eur Neurol 74: 243250 . 